\title{
Oportunismo alimentar de Knodus moenkhausii (Teleostei, Characidae): uma espécie abundante em riachos do noroeste do Estado de São Paulo, Brasil
}

\author{
Mônica Ceneviva-Bastos \& Lilian Casatti
}

Laboratório de Ictiologia, Departamento de Zoologia e Botânica, IBILCE, Universidade Estadual Paulista (UNESP), Rua Cristóvão Colombo, 2265, Jardim Nazareth, 15054-000, São José do Rio Preto, SP, Brasil, 0-55-17-32212396. (MCBastos@gmail.com, lcasatti@ibilce.unesp.br)

\begin{abstract}
Feeding opportunism of Knodus moenkhausii (Teleostei, Characidae): an abundant species in streams of the northwestern in the state of São Paulo, Brazil. In the present investigation we studied the feeding biology of Knodus moenkhausii (Eigenmann \& Kennedy, 1903) in streams of the Upper Paraná river system in the state of São Paulo. In eight streams (R1-R8), K. moenkhausii consumed 18 items, of which algae, ephemeropteran nymphs, and dipteran larvae were the most frequent and dominant autochthonous items, as were terrestrial insects, hymenopterans, and spiders regarding allochthonous items. In the R2 stream, $K$. moenkhausii showed distinct diet, mostly due to depth, substrate type, and presence of riparian vegetation. In the R9 stream (monthly sampled for one year), 15 items were consumed, of which terrestrial insects predominated all year round; dipteran larvae and algae were least relevant during December-January (hot and wet period) and June-July (cold and dry period), respectively. During snorkeling observations in this stream, we recorded individuals performing drift feeding, picking at relatively small preys close to the substrate and submerged vegetation, and surface picking. The large diversity of consumed items - conditioned to habitat and seasonal variations - and the diversity of feeding tactics allow us to consider $K$. moenkhausii a rather opportunistic fish species. Such opportunism apparently reflects on the species abundance, showing its ability to allocate a significant part of its energy to reproduction, even in physically impacted habitats by human actions.
\end{abstract}

KEYWORDS. Nektonics, omnivory, feeding tactics, seasonality.

RESUMO. O objetivo deste estudo foi investigar a biologia alimentar de Knodus moenkhausii (Eigenmann \& Kennedy, 1903) em riachos do Alto rio Paraná no Estado de São Paulo. Em oito riachos (R1-R8), K. moenkhausii se alimentou de 18 itens, dos quais algas, ninfas de efemerópteros e larvas de dípteros foram os itens autóctones mais freqüentes e dominantes; fragmentos de insetos terrestres, himenópteros e aranhas foram os itens alóctones mais freqüentes e dominantes. No riacho R2, K. moenkhausii apresentou dieta distinta dos demais riachos, principalmente em função da profundidade, tipo de substrato e da presença de vegetação ripária. No riacho R9, amostrado mensalmente durante um ano, foram identificados 15 itens, dos quais insetos terrestres predominaram ao longo do ano; larvas de dípteros e algas foram pouco expressivas nos períodos de dezembro-janeiro (período mais quente e chuvoso) e junho-julho (período mais frio e seco). No riacho R9 foram realizadas observações subaquáticas durante mergulho livre, onde observamos a cata de itens na coluna d'água junto do substrato, da vegetação submersa e na superfície da água. A elevada variedade de itens consumidos - condicionada às variações do hábitat e sazonais - e a prática de diversas táticas nos permitem considerar $K$. moenkhausii uma espécie oportunista quanto ao uso dos recursos alimentares. Este oportunismo aparentemente se reflete na abundância da espécie, demonstrando boa capacidade em alocar parte significativa de sua energia à reprodução, mesmo em ambientes fisicamente impactados por ação antrópica.

PALAVRAS-CHAVE. Nectônicos, onivoria, táticas alimentares, sazonalidade.

Os peixes de água doce neotropicais representam um oitavo da diversidade de vertebrados viventes e constituem a maior de todas as faunas epicontinentais do planeta (VARi \& Malabarba, 1998). A família mais representativa em termos de número de espécies é Characidae, com pelo menos 1.352 espécies (Reis et al., 2003). Este grupo forma o principal conjunto de espécies nectônicas em riachos de médio e baixo gradiente (BuCKuP, 1999), predominando inclusive em termos de abundância e biomassa (vide CASTRo et al., 2003, 2004, 2005) e desempenhando um papel importante na produção de peixes de riachos. Apesar dessa importância, pouco se conhece a respeito da ecologia das espécies de Characidae, especialmente sobre como alterações de origem antrópica nos ecossistemas podem influenciar a utilização de recursos alimentares e a sobrevivência dessas espécies.

No sistema do Alto rio Paraná, particularmente em drenagens afluentes da margem esquerda do rio Paraná no noroeste e oeste do Estado de São Paulo, amostragens recentes em 59 riachos (CASTRo et al., 2005; CASATTI et al., 2006) registraram que Astyanax altiparanae Garutti \& Britski, 2000 e Knodus moenkhausii (Eigenmann \& Kennedy, 1903) estão entre as espécies dominantes, sendo que a última representou $12 \%$ da abundância total de peixes coletados. Apesar da evidente posição de destaque que esta espécie ocupa em termos de estrutura quantitativa das comunidades, informações sobre sua biologia são desconhecidas. Neste trabalho, investigamos a biologia alimentar de K. moenkhausii, procurando responder (1) quais os itens alimentares preferenciais, (2) se a dieta varia conforme características do hábitat, (3) se há influência da sazonalidade na dieta da espécie e (4) quais as principais táticas alimentares empregadas na captura de alimento.

\section{MATERIAL E MÉTODOS}

A área de estudo situa-se no noroeste do Estado de São Paulo (Fig. 1), em uma região onde o solo é 
principalmente utilizado para pastagens $(77,4 \%)$ (Alexandre M. Silva, com. pess.). O clima da região é tropical quente (NIMER, 1989), com uma estação chuvosa geralmente de outubro a março, com temperatura média máxima $\left(31^{\circ} \mathrm{C}\right)$ em janeiro, e outra seca, com temperatura média mínima $\left(13^{\circ} \mathrm{C}\right)$ registrada em julho (BARCHA \& ARID, 1971). Com o objetivo de investigar se a dieta de $K$. moenkhausii varia conforme as características do hábitat, oito riachos (R1-R8, Tab. I) foram amostrados em junho de 2003, utilizando-se duas passagens de pesca elétrica (metodologia modificada a partir de MAZzONI et al., 2000 e CAStro et al., 2003). Para investigar a influência da sazonalidade na dieta, coletas mensais no período de agosto de 2003 a julho de 2004 foram conduzidas no Córrego do Cedro (R9, Tab. I), município de Vitória Brasil, através da aplicação de um esforço padronizado de coleta com rede de arrasto. Todos os peixes coletados foram fixados em formalina a $10 \%$ e posteriormente conservados em solução de etanol a 70\%. Exemplares-testemunho estão depositados na coleção ictiológica do Departamento de Zoologia e Botânica da Universidade Estadual Paulista, campus de São José do Rio Preto, SP (DZSJRP 6065, 6079, 6089, 6098, 6131, 6144, 6163, 6183).

Dez exemplares adultos dos riachos R1-R8, somando 80 indivíduos, mais 120 exemplares coletados no riacho R9 (dez indivíduos adultos selecionados por mês) tiveram seus estômagos removidos e seus conteúdos analisados e identificados no menor nível taxonômico possível. Especialistas foram consultados para assegurar a confiabilidade das determinações taxonômicas.

Para cada item foi calculada a freqüência de ocorrência (GELwick \& MATTHEws, 1996) e a dominância (Frost \& Went, 1940 apud Hynes, 1950), esta dada como a porcentagem do número de vezes em que o item ocupa a maior parte do conteúdo de cada estômago em função do número total de exemplares analisados. A composição percentual (GELWICK \& MATTHEWS, 1996) foi calculada para os itens alimentares agrupados nas seguintes categorias: algas, larvas e ninfas aquáticas de insetos, microcrustáceos, vegetais superiores, aracnídeos, insetos terrestres, peixes, matéria orgânica e itens não determinados. A amplitude de nicho trófico no riacho R9 foi calculada a partir da fórmula proposta por LEVINS (1968): B = $1 / \mathrm{p}_{\mathrm{i}}^{2}$, onde "B" é a amplitude de nicho e " $p$ ", é a proporção do recurso " $\mathrm{i}$ ". O valor de "B" obtido foi convertido para a escala de 0 a 1 , conforme proposto por HURLBERT (1978).

A comparação da dieta de $K$. moenkhausii entre os riachos R1-R8, bem como entre distintas épocas do ano no riacho $\mathrm{R} 9$, foi conduzida através de uma análise de escalonamento multidimensional não-métrica (NMDS), uma técnica de ordenação que pode ser aplicada a várias situações ecológicas, cujo cálculo é baseado em uma matriz de dissimilaridade ou de similaridade (CLARKE \& WARWICK, 1994). Os valores de composição percentual dos itens agrupados em categorias ecológicas foram transformados em $\log (\mathrm{x}+1)$ para cálculo da matriz de similaridade através do coeficiente de similaridade de Bray-Curtis, utilizando-se o programa estatístico PRIMER (CLARKE \& Gorley, 2001). A NMDS calcula a distorção, ou stress, entre a matriz de similaridade e a ordenação produzida na representação gráfica dos eixos. No presente estudo, todos os valores de stress estiveram abaixo de 0,1 , o que corresponde a uma boa ordenação, garantindo boa confiabilidade na interpretação dos resultados (CLARKE \& WARWICK, 1994).

Duas hipóteses nulas (Ho) foram testadas através de análises de similaridade (ANOSIM): (1) os descritores do hábitat não exercem influências significativas na dieta de K. moenkhausii nos riachos R1-R8; (2) não há diferenças significativas na dieta de $K$. moenkhausii entre distintas épocas do ano no riacho R9. A ANOSIM é uma análise não paramétrica, análoga à análise de variância (Clarke \& W ARWick, 1994), e foi processada através do programa computacional PRIMER (CLARKE \& GORLEY, 2001). Nesta análise, calcula-se o valor de R, uma medida

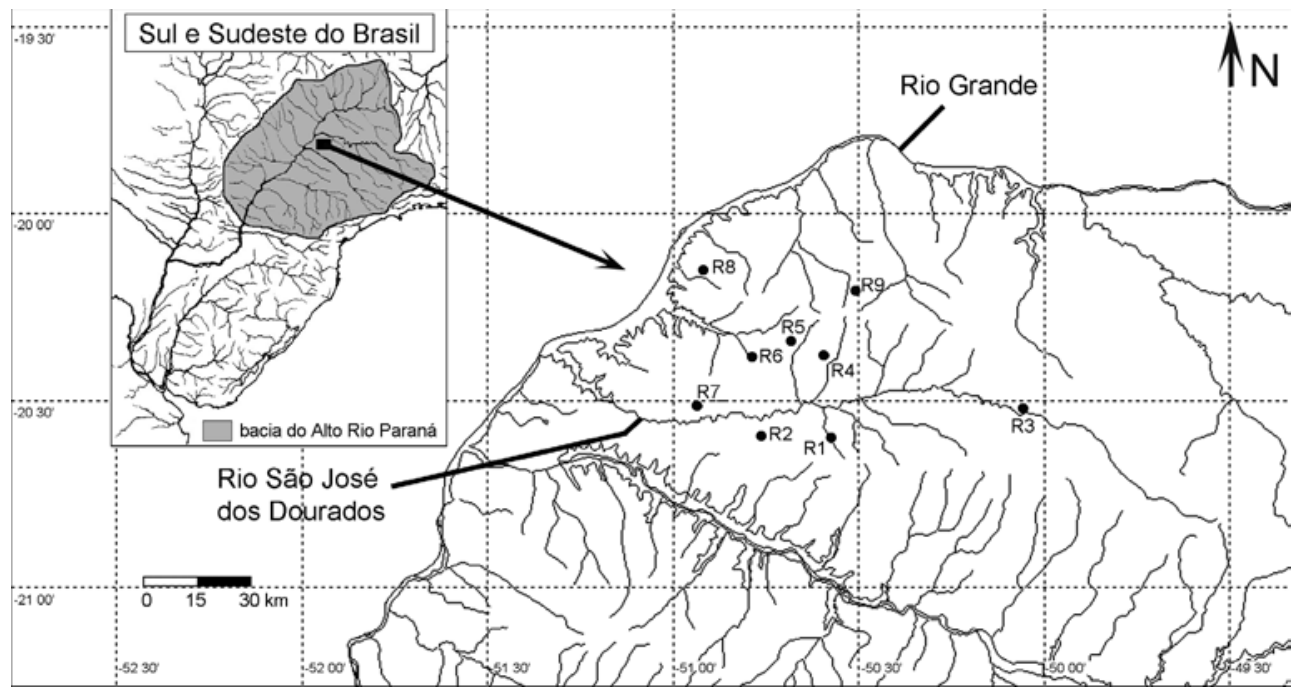

Fig. 1. Localização geográfica dos nove riachos estudados (R1-R9) na porção noroeste do Estado de São Paulo, sistema do Alto rio Paraná (Brasil). 
comparativa do grau de separação das amostras, que irá permear a decisão de aceite ou rejeição de Ho. A amplitude de variação de $\mathrm{R}$ é de -1 a 1 , sendo que valores próximos a zero significam aceite de $\mathrm{Ho}$, e a probabilidade (p) representa a porcentagem de risco de ocorrer erro Tipo I, segundo a nomenclatura estatística (CLARKE \& WARWICK, 1994).

Com o objetivo de explorar se há correlação significativa entre as características do hábitat e a dieta de K. moenkhausii, foi construída uma matriz com os itens alimentares agrupados nas categorias descritas acima e outra com os descritores ambientais, alguns diretamente mensurados, tais como a velocidade média da corrente e profundidade média, outros categorizados a partir de estimativas visuais, tais como: presença ou ausência de vegetação ripária (1, ausente; 2 , presente), tipo de substrato predominante (1, areia; 2 , argila; 3 , cascalho e rochas), tipos de hábitats fluviais predominantes (de acordo com Rincón, 1999, as principais categorias de hábitats em riachos são "riffles", "pools" e "runs", os dois primeiros equivalentes a corredeiras e poços; na ausência de um termo apropriado em português para se referir às regiões com águas correntes, porém mais profundas que corredeiras, empregaremos o termo em inglês) (1, somente "runs"; 2, "runs" e poços; 3, "runs", poços e corredeiras). As duas matrizes foram confrontadas através do teste de Mantel (MANTel, 1967), utilizando a rotina Matrix Comparison do programa computacional NTSyspc 2.10 (RoHLF, 2000), sendo que para a matriz alimentar foi utilizado o coeficiente de BrayCurtis e para a matriz ambiental, a Distância Euclidiana.

Para o registro das táticas alimentares foram conduzidos 145 minutos de observação subaquática durante mergulho livre no riacho R9, em outubro de 2004, empregando a técnica "animal focal" (Altmann, 1974). Também foram registrados o número de indivíduos, a posição e orientação dos mesmos na coluna d'água, tipo de substrato e micro-hábitats de forrageamento. A classificação das táticas alimentares seguiu KeENLEYSIDE (1979), SAZIMA (1986) e GRANT \& NOAKES (1987).

\section{RESULTADOS}

Nos riachos R1 a R8, K. moenkhausii se alimentou de 18 itens, dos quais algas do gênero Spyrogira, ninfas de efemerópteros e larvas de dípteros (principalmente Chironomidae e Simulidae) foram os itens autóctones mais freqüentes e dominantes; fragmentos de insetos terrestres, himenópteros (especialmente Formicidae) e aranhas foram os itens alóctones mais freqüentes e dominantes (Tab. II). Em termos de composição percentual, insetos terrestres $(26 \%)$ e larvas e ninfas aquáticas de insetos $(27 \%)$ tiveram maior representatividade, seguidos de itens de origem desconhecida (20\%). Estes foram representados por ovos de invertebrados e matéria orgânica, cujo grau de digestão e fragmentação impossibilitou uma identificação mais precisa, ou seja, se eram de origem animal ou vegetal, ou de origem autóctone ou alóctone.

A ordenação produzida através da NMDS mostra que no riacho R2 (o mais raso e o único a apresentar vegetação ripária e com substrato predominantemente argiloso), K. moenkhausii apresentou dieta relativamente distinta daquela encontrada nos demais riachos (Fig. 2). Os indivíduos analisados de R2 não apresentaram algas em seus estômagos (Fig. 3), ao contrário de aracnídeos, que foram relativamente abundantes (Fig. 4). Insetos terrestres, representados principalmente por Formicidae, apresentaram expressiva contribuição à dieta de $K$. moenkhausii na maioria dos riachos estudados (Fig. 5). De acordo com a estatística de Mantel, a dieta mostrou correlação significativa com os descritores do hábitat ( $\mathrm{r}$ $=0,5835, \mathrm{p}=0,001)$, sendo que aqueles que mais

Tabela I. Localização e descritores do hábitat dos riachos estudados na porção noroeste do Estado de São Paulo, Brasil.

\begin{tabular}{|c|c|c|c|c|c|c|}
\hline Riachos & $\begin{array}{l}\text { Coordenadas } \\
\text { geográficas }\end{array}$ & $\begin{array}{l}\text { Velocidade média } \\
\text { da água }(\mathrm{cm} / \mathrm{s})\end{array}$ & $\begin{array}{l}\text { Profundidade } \\
\text { média }(\mathrm{cm})\end{array}$ & $\begin{array}{l}\text { Vegetação } \\
\text { ripária }\end{array}$ & $\begin{array}{c}\text { Substrato } \\
\text { predominante }\end{array}$ & $\begin{array}{c}\text { Hábitats fluviais } \\
\text { predominantes }\end{array}$ \\
\hline $\mathrm{R} 1$ & $\begin{array}{l}50^{\circ} 34^{\prime} 05,0{ }^{\prime} \mathrm{W} \\
20^{\circ} 36^{\prime} 20,77^{\prime} \mathrm{S}\end{array}$ & 19 & 30 & ausente & areia & "runs" e poços \\
\hline $\mathrm{R} 2$ & $\begin{array}{l}50^{\circ} 45^{\prime} 29,2{ }^{\prime \prime} \mathrm{W} \\
20^{\circ} 35^{\prime} 49,5 ” \mathrm{~S}\end{array}$ & 26 & 20 & presente & argila consolidada & $\begin{array}{c}\text { "runs" e } \\
\text { corredeiras }\end{array}$ \\
\hline R3 & $\begin{array}{l}51^{\circ} 02 ' 56,4 ” \mathrm{~W} \\
20^{\circ} 31^{\prime} 28,8 ” \mathrm{~S}\end{array}$ & 24 & 70 & ausente & areia & "runs" e poços \\
\hline $\mathrm{R} 4$ & $\begin{array}{l}50^{\circ} 35^{\prime} 08,6 ” \mathrm{~W} \\
20^{\circ} 22^{\prime} 58,6 ” \mathrm{~S}\end{array}$ & 58 & 70 & ausente & areia & $\begin{array}{c}\text { "runs", poços e } \\
\text { corredeiras }\end{array}$ \\
\hline R5 & $\begin{array}{l}50^{\circ} 40^{\prime} 42,6 ” \mathrm{~W} \\
20^{\circ} 20^{\prime} 51,3 ” \mathrm{~S}\end{array}$ & 15 & 100 & ausente & areia & "runs" e poços \\
\hline R6 & $\begin{array}{l}50^{\circ} 46 ' 54,3 ” \mathrm{~W} \\
20^{\circ} 23^{\prime} 11,3 ” \mathrm{~S}\end{array}$ & 9 & 90 & ausente & areia & "runs" e poços \\
\hline R7 & $\begin{array}{l}50^{\circ} 55^{\prime} 37,1{ }^{\prime \prime} \mathrm{W} \\
20^{\circ} 31^{\prime} 08,1 ” \mathrm{~S}\end{array}$ & 45 & 60 & ausente & $\begin{array}{l}\text { areia, rochas e } \\
\text { cascalho }\end{array}$ & $\begin{array}{c}\text { "runs", poços e } \\
\text { corredeiras }\end{array}$ \\
\hline $\mathrm{R} 8$ & $\begin{array}{l}50^{\circ} 54{ }^{\prime} 46,5 " \mathrm{~W} \\
20^{\circ} 09^{\prime} 15,9 " \mathrm{~S}\end{array}$ & 8 & 60 & ausente & areia & "runs" e poços \\
\hline R9 & $\begin{array}{l}50^{\circ} 30 ’ 09,9 " \mathrm{~W} \\
20^{\circ} 12 ' 43,3 ” \mathrm{~S}\end{array}$ & 4 & 49 & ausente & areia & "runs" \\
\hline
\end{tabular}


influenciaram tal correlação foram profundidade $(r=0,38$, $\mathrm{p}<0,2)$, tipo de substrato predominante $(\mathrm{r}=0,31, \mathrm{p}<0,2)$ e, principalmente, presença/ausência de vegetação ripária $(\mathrm{r}=0,89, \mathrm{p}<0,1)$.

No riacho R9 foram identificados 15 itens (Tab. III), tanto alóctones como autóctones, com predominância de insetos terrestres (principalmente Formicidae), larvas de dípteros (principalmente Chironomidae) e algas (principalmente Spyrogira). Como pode ser visto na Fig. 6 , a dieta de $K$. moenkhausii foi mais dissimilar nos meses de menor temperatura e pluviosidade (junho-julho), diferença esta também apontada pela estatística $\mathrm{R}$ calculada através da ANOSIM $(R=0,67, p<0,2)$. Algas constituíram um item especialmente pouco expressivo nos meses de menor temperatura e pluviosidade (junho-julho, Fig. 7), enquanto que as larvas e ninfas aquáticas de insetos foram pouco representativas nos meses de maior temperatura e pluviosidade (dezembro-janeiro, Fig. 8). Vegetais superiores foram mais representativos nos períodos de temperatura e pluviosidade intermediárias (Fig. 9).

A menor amplitude de nicho trófico $(\mathrm{Ba}=0,53)$ foi registrada no período de maior temperatura e pluviosidade (dezembro-janeiro), enquanto que a maior $(\mathrm{Ba}=0,85)$ foi registrada no período de menor temperatura e pluviosidade (junho-julho). Além disso, não foram registradas diferenças significativas entre a proporção de itens autóctones e alóctones entre os períodos de maior e menor pluviosidade (autóctones: $\chi^{2}=0, p=1,000$; alóctones: $\chi^{2}=$ $0,627, \mathrm{p}=0,4882$ ).

Grupos de 5-20 indivíduos de K. moenkhausii foram observados forrageando continuamente, praticando a cata de itens na coluna d'água carregados pela correnteza ("drift feeding", cf. GRANT \& NOAKES, 1987), a cata de itens junto ao substrato ou à vegetação submersa ("picking at relatively small prey", cf. KeENLEyside, 1979) e a cata de itens na superfície da água ("surface picking", cf. SAZIMA, 1986) (Fig. 10). Enquanto praticavam a cata de itens arrastados pela correnteza, os peixes nadavam orientados contra a correnteza; após inspecionarem o item (podendo ou não capturá-1o), deixavam-se levar pela correnteza, para novamente nadar rio acima. Indivíduos de pequeno porte formaram grupos menores (até oito indivíduos), espacialmente restritos às áreas mais rasas, próximas às margens (Fig. 10), investindo em porções mais profundas do riacho apenas na ausência de adultos.

\section{DISCUSSÃO}

Vários estudos indicam que fontes alimentares alóctones parecem ser de maior relevância na dieta de peixes de riachos (p. ex., SABINO \& CASTRO, 1990; WALKER, 1990; Sabino \& Zuanon, 1998; Esteves \& Lobón-Cerviá, 2001). Em contrapartida, estudos como os de UiEDA et al. (1997) e CASATTI (2002) revelaram que não há predomínio

Tabela II. Número de exemplares coletados, média e desvio padrão do comprimento padrão (CP em mm) e itens alimentares consumidos por Knodus moenkhausii nos riachos R1-R8, sistema do Alto rio Paraná (SP, Brasil), com seus respectivos valores de freqüência de ocorrência $(\mathrm{FO}, \%)$ e dominância $(\mathrm{D}, \%)$. Traços indicam que, apesar de presente, o item não foi dominante.

\begin{tabular}{|c|c|c|c|c|c|c|c|c|c|c|c|c|c|c|c|c|}
\hline \multirow{4}{*}{$\begin{array}{l}\mathrm{N} \\
\mathrm{CP} \text { (média } \pm \text { desvio padrão) }\end{array}$} & \multirow{2}{*}{\multicolumn{2}{|c|}{$\begin{array}{l}\mathrm{R} 1 \\
29\end{array}$}} & \multicolumn{2}{|c|}{$\mathrm{R} 2$} & \multicolumn{2}{|c|}{ R3 } & \multicolumn{2}{|c|}{ R4 } & \multicolumn{2}{|c|}{ R5 } & \multicolumn{2}{|c|}{ R6 } & \multicolumn{2}{|c|}{$\mathrm{R} 7$} & \multicolumn{2}{|c|}{$\mathrm{R} 8$} \\
\hline & & & \multirow{2}{*}{\multicolumn{2}{|c|}{$\begin{array}{c}241 \\
17,7 \pm 4,9\end{array}$}} & \multicolumn{2}{|c|}{188} & \multicolumn{2}{|c|}{225} & \multicolumn{2}{|c|}{103} & \multicolumn{2}{|c|}{29} & \multicolumn{2}{|c|}{33} & \multicolumn{2}{|c|}{24} \\
\hline & \multicolumn{2}{|c|}{$29,7 \pm 6,5$} & & & 24,6 & $\pm 4,0$ & 18,2 & $\pm 4,5$ & 28,0 & $=4,0$ & $27,0=$ & $=5,0$ & 31,6 & $\pm 4,2$ & $25,3 \pm$ & 10,2 \\
\hline & FO & $\mathrm{D}$ & FO & $\mathrm{D}$ & FO & $\mathrm{D}$ & FO & $\mathrm{D}$ & FO & $\mathrm{D}$ & FO & $\mathrm{D}$ & $\mathrm{FO}$ & $\mathrm{D}$ & FO & $\mathrm{D}$ \\
\hline \multicolumn{17}{|l|}{ ITENS AUTÓCTONES } \\
\hline Algas & 60 & 30 & & & 40 & 40 & 40 & - & 20 & 10 & 60 & 40 & 67 & 40 & 100 & 90 \\
\hline Larvas de dípteros & 50 & 10 & 60 & - & 10 & 10 & 40 & - & & & 10 & - & 44 & - & 40 & - \\
\hline Larvas de simulídeos & 30 & 20 & 50 & 10 & 10 & - & 30 & - & & & & & 22 & - & 30 & - \\
\hline Outras larvas de insetos & 10 & - & & & & & & & & & 10 & - & 11 & 10 & 20 & - \\
\hline Ninfas de efemerópteros & 50 & 10 & 70 & - & & & 10 & 10 & & & 10 & 10 & 22 & 10 & 20 & - \\
\hline Pupas de dípteros & 10 & 10 & & & & & 10 & 10 & & & & & 11 & - & & \\
\hline Microcrustáceos & 10 & - & & & & & & & & & & & & & & \\
\hline \multicolumn{17}{|l|}{ ITENS ALÓCTONES } \\
\hline Vegetais superiores & & & 30 & - & & & 60 & 10 & 10 & - & 20 & - & 44 & - & & \\
\hline Hemípteros & 10 & - & 10 & 10 & & & & & & & & & & & & \\
\hline Aranhas / ácaros & & & 50 & 10 & & & & & 10 & 10 & 10 & - & 22 & - & 30 & - \\
\hline Isópteros & & & 80 & 10 & & & & & & & & & & & & \\
\hline Coleópteros adultos & & & 30 & 20 & 20 & 20 & 30 & 10 & & & 10 & 10 & - & - & & \\
\hline Himenópteros & & & 40 & 10 & 20 & 10 & 30 & 20 & 30 & 10 & 20 & 10 & 11 & - & 10 & - \\
\hline Outros invertebrados & 10 & - & 40 & - & 10 & - & 20 & - & 10 & 10 & - & - & 11 & - & 10 & - \\
\hline Fragmentos de insetos & 50 & - & 50 & 20 & 40 & 20 & 50 & 20 & 60 & 30 & 60 & 20 & 67 & 20 & 60 & - \\
\hline \multicolumn{17}{|l|}{ ORIGEM DESCONHECIDA } \\
\hline Ovos & 10 & - & 10 & - & & & & & 40 & 30 & 10 & - & & & & \\
\hline Fragmentos não identificados & & & 40 & 10 & & & 10 & 10 & & & & & 11 & 10 & 10 & - \\
\hline Matéria orgânica & 70 & 20 & & & 50 & - & 70 & 10 & 30 & - & 70 & 10 & 44 & - & 80 & 10 \\
\hline Matéria inorgânica & & & & & & & & & & & 20 & - & 22 & - & 10 & - \\
\hline
\end{tabular}




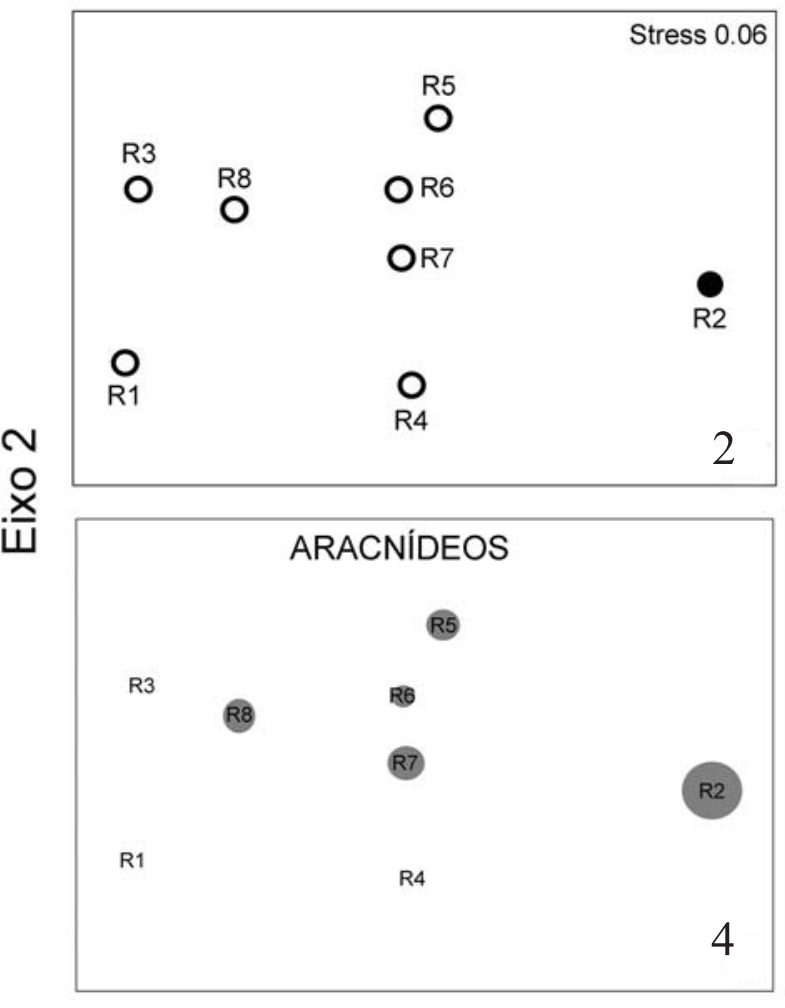

\section{Eixo 1}

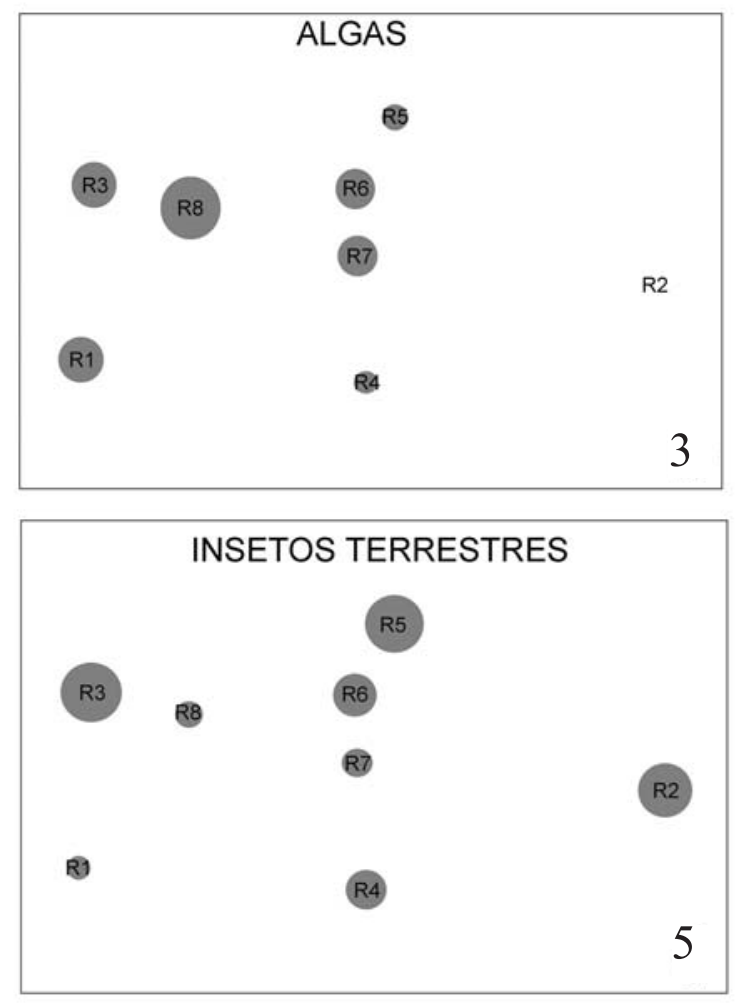

\section{Eixo 1}

Figs. 2-5. 2, ordenação resultante da análise de escalonamento multidimensional não métrica (NMDS) sobre a dieta de Knodus moenkhausii em oito riachos (R1-R8) no sistema do Alto rio Paraná, SP, Brasil; círculos vazios representam riachos sem vegetação ripária e com substrato predominantemente arenoso, círculo cheio representa riacho com vegetação ripária, raso e com substrato argiloso. 3-5, contribuição (expressa conforme o tamanho do círculo) de algas, aracnídeos e insetos terrestres na dieta de Knodus moenkhausii nos oito riachos estudados.

Tabela III. Itens alimentares consumidos mensalmente por Knodus moenkhausii no riacho R9, sistema do Alto rio Paraná (SP, Brasil) no período de agosto de 2003 a julho de 2004, com seus respectivos valores de frequiência de ocorrência (FO, \%) e dominância (D, \%). Traços indicam que, apesar de presente, o item não foi dominante.

\begin{tabular}{|c|c|c|c|c|c|c|c|c|c|c|c|c|c|c|c|c|c|c|c|c|c|c|c|c|}
\hline & \multicolumn{2}{|c|}{ A } & \multicolumn{2}{|c|}{$\mathrm{S}$} & \multicolumn{2}{|c|}{$\mathrm{O}$} & \multicolumn{2}{|c|}{$\mathrm{N}$} & \multicolumn{2}{|c|}{$\mathrm{D}$} & \multicolumn{2}{|c|}{$\mathrm{J}$} & \multicolumn{2}{|c|}{$\mathrm{F}$} & \multicolumn{2}{|c|}{ M } & \multicolumn{2}{|c|}{ A } & \multicolumn{2}{|c|}{ M } & \multicolumn{2}{|c|}{$\mathrm{J}$} & \multicolumn{2}{|l|}{$\mathrm{J}$} \\
\hline & FO & $\mathrm{D}$ & $\mathrm{FO}$ & $\mathrm{D}$ & $\mathrm{FO}$ & $\mathrm{D}$ & FO & $\mathrm{D}$ & FO & $\mathrm{D}$ & FO & $\mathrm{D}$ & $\mathrm{FO}$ & $\mathrm{D}$ & $\mathrm{FO}$ & $\mathrm{D}$ & $\mathrm{FO}$ & $\mathrm{D}$ & FO & $\mathrm{D}$ & FO & $\mathrm{D}$ & $\mathrm{FO}$ & $\mathrm{D}$ \\
\hline \multicolumn{25}{|l|}{ ITENS AUTÓCTONES } \\
\hline Algas & 90 & 50 & 40 & 10 & 60 & 20 & 60 & 10 & 55,61 & 11,1 & 70 & 10 & 60 & 10 & 60 & 10 & 20 & 10 & 70 & 30 & 20 & 10 & 40 & - \\
\hline Larvas de dípteros & 50 & - & 60 & 10 & 50 & - & 70 & 10 & 22,2 & - & 40 & - & 50 & - & 40 & - & 80 & 40 & 40 & - & 50 & 10 & 40 & 10 \\
\hline Outras larvas de insetos & 20 & - & 30 & - & 10 & - & 20 & - & 11,1 & - & 10 & - & 10 & - & & & 10 & - & 10 & - & & & 10 & - \\
\hline Ninfas de efemerópteros & 30 & 20 & 20 & - & 10 & - & 10 & - & & & & & 10 & - & & & 50 & 30 & 20 & 20 & 10 & - & 10 & 10 \\
\hline Pupas de dípteros & 10 & - & & & & & & & & & & & & & & & & & & & & & & \\
\hline Peixe & & & & & & & & & & & & & & & & & 10 & 10 & & & & & & \\
\hline \multicolumn{25}{|l|}{ ITENS ALÓCTONES } \\
\hline Vegetais superiores & 50 & - & 20 & - & 10 & - & 40 & 10 & & & 20 & 10 & 60 & 20 & 50 & 10 & 20 & - & 60 & 20 & 10 & - & 20 & 10 \\
\hline Aranhas / ácaros & & - & 10 & - & & & & & & & 10 & - & 10 & - & 10 & - & 10 & - & & & & & & \\
\hline Dípteros adultos & & & & - & & & & & & & 10 & 10 & & & 20 & - & & & 10 & 10 & & & & \\
\hline Coleópteros adultos & 10 & - & & - & & & & & & & & & 20 & 10 & 10 & 10 & & & & & & & & \\
\hline Himenópteros & 20 & - & & - & 40 & 40 & 40 & 10 & 33,33 & 33,3 & & & 30 & 20 & 40 & 20 & 10 & - & 10 & 10 & 20 & 10 & & \\
\hline Fragmentos de insetos & 60 & - & 100 & 40 & 60 & 40 & 60 & 40 & 33,32 & 22,2 & 90 & - & 100 & 40 & 60 & 10 & 30 & - & 80 & - & 80 & 30 & 20 & 10 \\
\hline \multicolumn{25}{|l|}{$\begin{array}{l}\text { ORIGEM } \\
\text { DESCONHECIDA }\end{array}$} \\
\hline Ovos & 20 & - & & & & & & & & & & & & & & & 10 & - & & & & & 30 & 20 \\
\hline $\begin{array}{l}\text { Fragmentos não } \\
\text { identificados }\end{array}$ & & & & & 10 & & & & & & & & & & 40 & 20 & 20 & 10 & 10 & - & 40 & 30 & 40 & 10 \\
\hline Matéria orgânica & 50 & - & 90 & 40 & 70 & - & 60 & 10 & 66,73 & 33,3 & 90 & 40 & 50 & - & 40 & 20 & 70 & - & 60 & 10 & 40 & 10 & 60 & 30 \\
\hline Matéria inorgânica & 30 & - & 30 & - & 10 & - & 60 & - & 44,4 & - & 90 & 30 & 20 & - & 40 & - & 20 & - & 80 & - & 10 & - & 30 & - \\
\hline
\end{tabular}


de itens autóctones ou alóctones para esses peixes. Explicar tal predominância freqüentemente envolve fatores em diversas escalas, tais como diversidade de hábitats, integridade da vegetação marginal, intensidade e tipo de ação antrópica aplicada à micro-bacia. Além disso, a abundância de itens autóctones muitas vezes depende dos nutrientes advindos da matéria orgânica proveniente da vegetação marginal, constituindo a base da cadeia trófica de pequenos riachos sombreados (Gregory et al., 1991), o que acentua ainda mais a importância da conservação da vegetação ripária para a biota local (ANGERMEIER \& KARR, 1983).

A diferença pouco expressiva entre o uso de recursos autóctones e alóctones encontrada tanto nos riachos R1-R8 quanto nas amostragens mensais de R9 é um sinal de que a espécie em questão muito provavelmente utilizaria um determinado tipo de item alimentar na ausência de outro de maior preferência. Muitas espécies de Characidae são oportunistas quanto ao uso de recursos alimentares em ambientes de riachos, conforme apontado por REZENDE \& MAZZONI (2003) e Mazzoni \& Rezende (2003) para Bryconamericus microcephalus (Miranda-Ribeiro, 1908) e Deuterodon sp., respectivamente.

A participação de itens autóctones ou alóctones na dieta de peixes de riachos pode variar quando investigada em termos da abundância relativa de cada espécie. Em um riacho da Mata Atlântica no sudeste do Brasil, Esteves \& LoBón-CERviÁ (2001) registraram que a maioria das espécies se alimenta de recursos autóctones, porém, quando considerada a abundância total, os itens alóctones passam a ser os mais importantes, sendo fundamentais para a manutenção de uma grande fração da biomassa de peixes neste local. Neste caso, a importância dos itens alóctones foi explicada pela dominância de Mimagoniates microlepis (Steindachner, 1876), uma espécie nectônica de Characidae, que eficientemente explora a superfície da água em poços, capturando insetos que caem da vegetação marginal (Costa, 1987; Aranha et al., 1998; Esteves \& LobÓNCerviá, 2001), da qual depende. No caso de $K$. moenkhausii, a participação praticamente eqüitativa de itens autóctones e alóctones sugere que essa espécie apresenta poucas exigências quanto à integridade da vegetação marginal para sua alimentação.

No riacho R2, a dieta de $K$. moenkhausii foi notadamente diferente dos demais (R1, R3-R8). Neste local, a vegetação ripária, apesar de degradada, está presente; o leito do riacho é predominantemente composto por argila consolidada e é o riacho mais raso dentre os analisados. Nos demais riachos, a vegetação marginal foi suprimida e praticamente todos se encontram em estágio avançado de assoreamento, com amplas áreas de deposição de areia. Assim, a dissimilaridade registrada

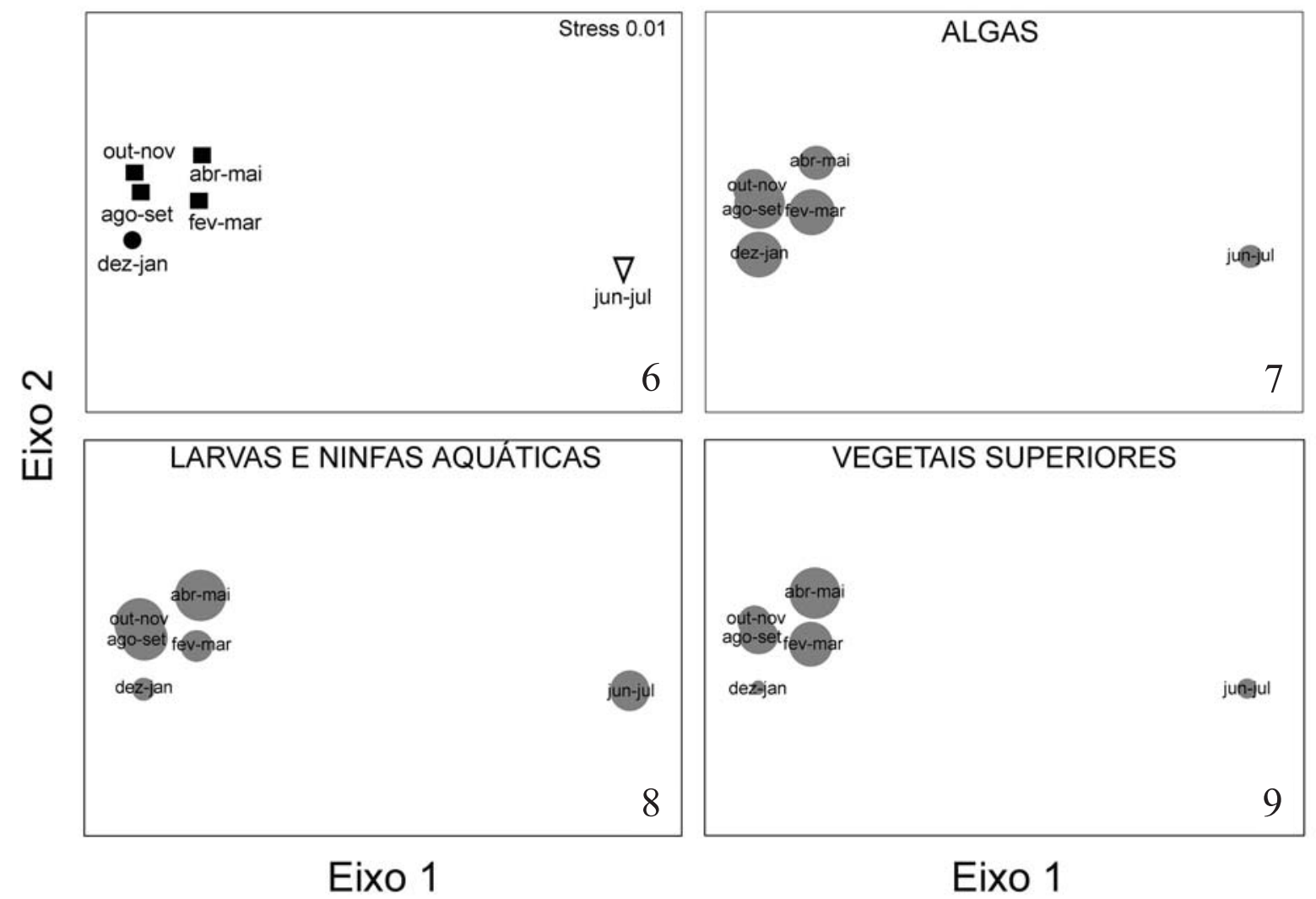

Figs. 6-9. 6, ordenação resultante da análise de escalonamento multidimensional não métrica (NMDS) sobre a dieta de Knodus moenkhausii no riacho R9 situado no sistema do Alto rio Paraná, SP, Brasil. Círculo representa meses com temperatura e pluviosidade elevadas; quadrados representam meses com temperatura e pluviosidade intermediárias; triângulo representa meses com temperatura e pluviosidade baixas. 7-9, contribuição (expressa conforme o tamanho do círculo) de algas, larvas e ninfas aquáticas de insetos e fragmentos de vegetais superiores na dieta de Knodus moenkhausii ao longo do período de amostragem. 
reflete maior disponibilidade de recursos alóctones, principalmente sementes, aracnídeos e insetos terrestres, que representaram 52,6\% da dieta. Em lagos de várzea da região Amazônica, CLARO-JR. et al. (2004) demonstraram que a alimentação de três espécies de peixes onívoros difere conforme o grau da ação antrópica na vegetação marginal, o que provavelmente se reflete em diferenças na disponibilidade dos recursos alimentares. Este conjunto de informações pode ser valioso quando incorporado a análises de integridade biótica especialmente porque, se comparadas com áreas de referência, a composição trófica de uma comunidade submetida a diferentes graus e tipos de interferência antrópica pode refletir alterações no transporte, transformação e armazenamento de energia (GOLDSTEIN $\&$ SimON, 1999).

Estudos sobre alimentação de peixes de riachos neotropicais indicam, em sua maioria, uma dieta euritrófica, que pode ser alterada frente a mudanças no ambiente decorrentes de ações antrópicas ou mesmo ao longo de flutuações sazonais (Lowe-McConnell, 1987; SABINo \& CAstro, 1990). Mudanças sazonais dos hábitos alimentares de Characidae têm sido amplamente registradas na literatura (p. ex., EsTEVES, 1996; LoBÓNCerviá \& Bennemann, 2000; Ortaz, 2001; Mazzoni \& Rezende, 2003). No riacho R9, os recursos que demonstraram maior influência da sazonalidade foram algas, insetos aquáticos e vegetais superiores, ao contrário de Formicidae e matéria orgânica, registrados ao longo do ano. De fato, Formicidae representa aproximadamente um terço da biomassa de insetos em regiões tropicais (HöLLDOBLER \& WILSON, 1990), constituindo um recurso alimentar abundante para determinadas guildas de peixes de riachos.

Algumas espécies de peixes onívoros podem apresentar dieta predominantemente invertívora durante a estação chuvosa e constituída basicamente por algas e detritos na estação seca, visto que estes dois últimos itens são geralmente abundantes em riachos (PREJS \& PREJS, 1987). Ao analisarem a dieta de Deuterodon iguape Eigenmann, 1907, uma espécie nectônica de Characidae, comum em riachos da Mata Atlântica, Deus \& PetrereJR. (2003) observaram que no verão houve maior consumo de detritos e algas enquanto que no inverno esta espécie consumiu basicamente algas, compondo um cenário oposto ao registrado no presente estudo. No riacho R9 observamos que algas e matéria orgânica foram pouco expressivas na dieta de $K$. moenkhausii durante o inverno, apesar de relativamente abundantes no verão. Tal resultado pode ser explicado pela queda da temperatura e luminosidade no inverno, que influencia negativamente o crescimento de algas verdes (ALLAN, 1995), como também pela influência de interações com outras espécies, tal como observado por Power (1983) em riachos no Panamá.

Durante o período mais chuvoso e quente foi verificada maior tendência à especialização alimentar, opondo-se ao registrado em alguns estudos realizados em riachos neotropicais (Prejs \& Prejs, 1987; Deus \& Petrere-JR., 2003). Se por um lado, a maior diversidade e abundância de recursos podem induzir ao uso oportuno dos mesmos, resultando num espectro alimentar mais generalista, por outro lado tendências à especialização podem ser explicadas pela escolha de itens energeticamente mais importantes, dentre os diversos oferecidos. Em contraste, a maior amplitude de nicho registrada na estação seca indica maior diversificação alimentar, o que é característico de espécies abundantes, adaptadas a ambientes instáveis (LEvins, 1968).

Nos estômagos provenientes do riacho R9, larvas e ninfas aquáticas de insetos foram mais abundantes no período mais seco e frio (junho-julho), ao contrário do observado nos meses mais chuvosos e quentes (dezembro-janeiro), quando estes itens foram raros. A baixa participação de macroinvertebrados aquáticos na dieta de espécies nectônicas durante o período mais chuvoso foi também constatada por ORTAZ (2001), em um riacho no norte da Venezuela. A redução na detecção das presas em função do aumento da turbidez da água e a influência da forte correnteza sobre os macroinvertebrados foram fatores apontados pelo autor para explicar esta observação (ORTAZ, 2001).

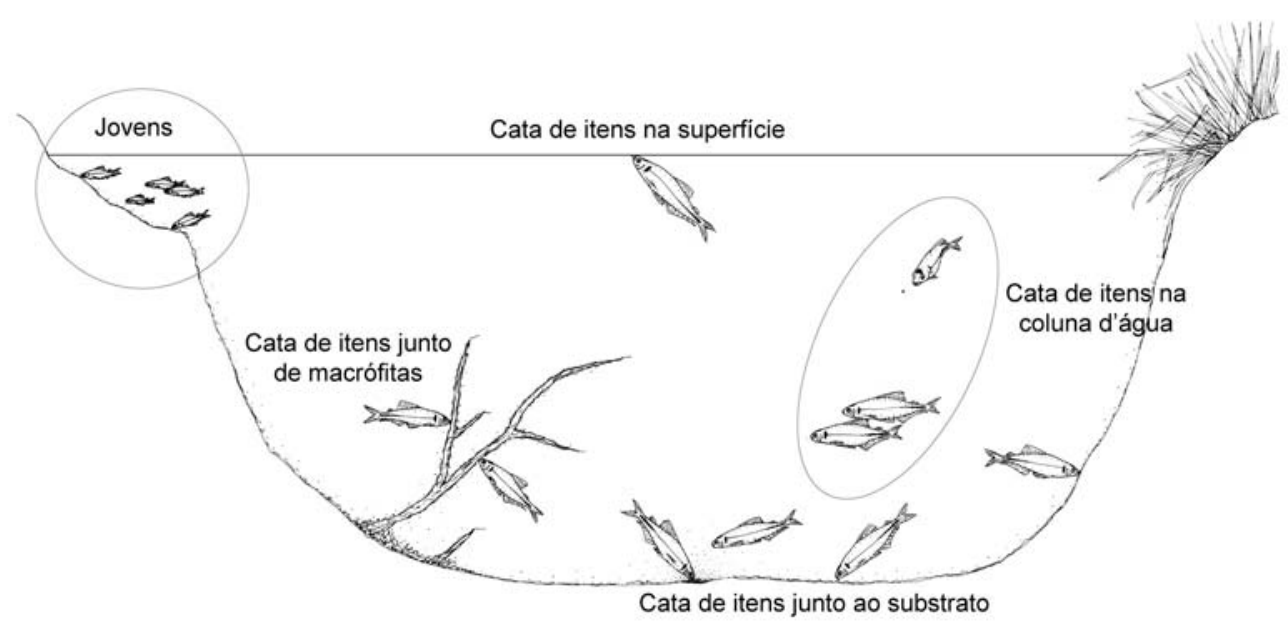

Fig. 10. Táticas alimentares e distribuição espacial durante o período de forrageamento de indivíduos jovens e adultos de Knodus moenkhausii observados no riacho R9 no sistema do Alto rio Paraná, SP, Brasil. 
A habilidade de alimentar-se de um número relativamente elevado de presas e a sazonalidade na dieta também foram registradas para espécies identificadas como K. deuterodonoides (Eigenmann, 1914) e Knodus sp., sintópicas em um riacho venezuelano (ORTAZ, 2001). As duas espécies apresentaram hábitos predominantemente invertívoros, consumindo maior diversidade de itens em sua maioria alóctones - na estação chuvosa. Além disso, a freqüência de estômagos vazios foi muito inferior à de repletos, tal como observamos no presente estudo (dois vazios dentre os 200 analisados). Tal fato pode ser explicado não somente pela provável abundância de recursos alimentares (ORTAZ, 2001), como também pela suposta eficácia na captura de alimento pela espécie. Tais hipóteses devem se aplicar à situação registrada no presente estudo, podendo ser futuramente testadas por meio de estudos sobre o comportamento alimentar e diversidade das presas em potencial.

Os caracídeos nectônicos de pequeno porte aparentemente apresentam dois padrões de ocupação de espaço para forrageamento: (1) espécies de pequeno porte, com corpos fusiformes, que formam cardumes numerosos, e são aparentemente mais especializadas na cata de itens arrastados pela correnteza ou na superfície da água (veja CAsATti \& CAstro, 1998, para Bryconamericus stramineus Eigenmann, 1908; SABINO \& ZuANON, 1998, para Bryconops giacopinni (FernandezYépez, 1950)); (2) espécies com corpos relativamente mais altos, que formam grupos menores, são mais oportunistas quanto ao emprego de táticas alimentares, e forrageiam em diversos estratos da coluna d'água e micro-hábitats (veja CASATTI \& CASTRO, 1998, para Astyanax rivularis (Lütken, 1874); Aranha et al., 1998, para Deuterodon langei Travassos, 1957 e Astyanax sp.), sendo este o padrão que mais se aplica a $K$. moenkhausii. Infelizmente, ainda são poucos os estudos que incorporam observações diretas no estudo da dieta de peixes de riachos (SABINO, 1999). Especialmente no noroeste do Estado de São Paulo, em função do elevado aporte de partículas causado pela remoção da vegetação ripária e uso da terra no entorno, são raros os ambientes onde a transparência da água favoreça o uso de mergulho livre. Apesar disso, temos observado que nos períodos de menor vazão, informações sobre táticas alimentares e distribuição espacial podem ser facilmente obtidas através deste método.

Casos de segregação espacial de acordo com tamanho corporal também foram registrados em outras espécies de Characidae em ambientes lóticos (CASATTi \& CAstro, 1998). Nossas análises, porém, não permitem sugerir se essa segregação reflete algum tipo de variação ontogenética na dieta, visto que apenas indivíduos adultos tiveram seus estômagos examinados. Ao analisar três espécies de Characidae em lagoas do sudeste do Brasil, Esteves (1996) observou diferenças ontogenéticas na dieta, atribuindo-as, em alguns casos, a mudanças no uso de micro-hábitats e nas táticas alimentares.

À medida que alterações antrópicas se intensificam em riachos, a estrutura trófica local pode ser alterada, de modo que as espécies que permanecem nestes ambientes são, na maioria das vezes, generalistas ou oportunistas. Estes termos são aplicados a espécies que exploram mais de uma guilda trófica, e refletem não somente o tipo de alimento ingerido, mas também estratégias alimentares e comportamentais de sobrevivência (GoLDSTEIN \& SiMON, 1999). Desta forma, a variedade de itens alimentares condicionada às variações sazonais do hábitat - e a prática de diversas táticas de captura de alimento, nos permite considerar K. moenkhausii uma espécie oportunista quanto ao uso dos recursos alimentares. Este oportunismo aparentemente se reflete na abundância da espécie, que demonstra boa capacidade em alocar parte significativa de sua energia à reprodução, o que a torna uma das espécies de peixes dominantes mesmo em ambientes fisicamente impactados por ação antrópica.

Agradecimentos. Agradecemos Fernando R. Carvalho, José L. Veronezi Jr., Fabíola C. Rocha, Daniel R. Lacerda, Danusa C. Ferreira e Renato M. Romero pela colaboração durante os trabalhos de campo; Francisco Langeani, Katiane M. Ferreira, Ricardo M. C. Castro e Richard P. Vari pelo auxílio na determinação taxonômica de Knodus moenkhausii; Fernando B. Noll, Reinaldo J. Feres e Orlando Necchi Jr. pelo auxílio com as determinações taxonômicas de insetos e algas; Rodrigo L. Moura e Tiago Gomes pela ajuda com programas de análises estatísticas; Denise de C. Rossa-Feres, Francisco Langeani, Cristiane P. Ferreira e revisores anônimos pelas valiosas sugestões dadas ao manuscrito; Departamento de Zoologia e Botânica IBILCE-UNESP pelo apoio; IBAMA pela permissão de coleta (001/2003). Este trabalho foi subvencionado pela Fundação de Amparo à Pesquisa do Estado de São Paulo (FAPESP), dentro do Programa BIOTA/FAPESP (www.biota.org.br) (01/13340-7, 02/05996-2, 03/12893-8).

\section{REFERÊNCIAS BIBLIOGRÁFICAS}

Allan, J. D. 1995. Stream ecology: structure and function of running waters. Dordrecht, Kluwer Academic. 388p.

Altmann, J. 1974. Observational study of behavior: sampling methods. Behaviour 49:227-265.

Angermeier, P. L. \& KARR, J. R. 1983. Fish communities along environmental gradients in a system of tropical streams. Environmental Biology of Fishes 9:117-135.

Aranha, J. M. R.; Takeuti, D. F. \& Yoshimura, T. M. 1998. Habitat use and food partioning of the fishes in a costal stream of Atlantic Forest, Brazil. Revista de Biología Tropical 46(4):951-959.

BArcha, S. F. \& ArID, F. M. 1971. Estudo de evapotranspiração na região Norte-Ocidental do Estado de São Paulo. Revista de Ciências 1:99-122.

Buckup, P. A. 1999. Sistemática e biogeografia de peixes de riachos. In: Caramaschi, E. P.; Mazzoni, R. \& Peres-Neto, P. R. eds. Ecologia de peixes de riachos. Série Oecologia Brasiliensis. Rio de Janeiro, PPGE-UFRJ. p.91-138.

Casatti, L. 2002. Alimentação dos peixes em um riacho do Parque Estadual Morro do Diabo, bacia do Alto Paraná, sudeste do Brasil. Biota Neotropica 2(2):1-14.

Casatti, L. \& Castro, R. M. C. 1998. Fish community of the São Francisco river headwaters riffles, southeastern Brazil. Ichthyological Exploration of Freshwaters 9(3):229-242.

Casatti, L.; Langeani, F.; Silva, A. M. \& Castro, R. M. C. 2006. Stream fish, water and habitat quality in a pasture dominated basin, southeastern Brazil. Brazilian Journal of Biology 66(2B):681-696

Castro, R. M. C.; Casatti, L.; Santos, H. F.; Ferreira, K. M.; Ribeiro, A. C.; Benine, R. C.; Dardis, G. Z. P.; Melo, A. L. A.; Stopiglia, R.; Abreu, T. X.; Bockmann, F. A.; Carvalho, M.; Gibran, F. Z. \& Lima, F. T. C. 2003. Estrutura e composição da ictiofauna de riachos do Rio Paranapanema, sudeste do Brasil. Biota Neotropica 3(1):1-31.

Castro, R. M. C.; Casatti, L.; Santos, H. F.; Melo, A. L. A.; Martins, L. S. F.; Ferreira, K. M.; Gibran, F. Z.; Benine, R. C.; Carvalho, M.; Ribeiro, A. C.; Abreu, T. X.; Bockmann, F. A.; Pelição, G. Z.; Stopiglia, R. \& Langeani, F. 2004. Estrutura e composição da ictiofauna de riachos da bacia do Rio Grande 
no Estado de São Paulo, sudeste do Brasil. Biota Neotropica 4(1): 1-39.

Castro, R. M. C.; Casatti, L.; Santos, H. F.; Vari, R. P.; Melo, A. L. A.; Martins, L. S. F.; Abreu, T. X.; Benine, R. C.; Gibran, F. Z.; Ribeiro, A. C.; Bockmann, F. A.; Carvalho, M.; Pelição, G. Z.; Ferreira, K. M.; Stopiglia, R. \& Akama, A. 2005. The stream ichthyofauna of four tributary rivers of the upper Rio Paraná basin, Brazil. Ichthyological Exploration of Freshwaters 16(3): 193-214

Clarke, K. R. \& Gorley, R. N. 2001. Software PRIMER v5. Plymouth, PRIMER-E. UK.

Clarke, K. R. \& Warwick, R. M. 1994. Change in marine communities: an approach to statistical analysis and interpretation. Plymouth, Plymouth Marine Laboratory. 144p.

Claro-Jr., L.; Ferreira, E.; Zuanon, J. \& Araújo-Lima, C. 2004. O efeito da floresta alagada na alimentação de três espécies de peixes onívoros em lagos de várzea da Amazônia Central, Brasil. Acta Amazonica 34(1):133-137.

Costa, W. J. E. M. 1987. Feeding habits of a fish community in a tropical coastal stream, Rio Mato Grosso, Brazil. Studies on Neotropical Fauna and Environment 22(3):145-153.

Deus, C. P. \& Petrere-Jr., M. 2003. Seasonal diet shifts of seven fish species in an Atlantic rainforest stream in Southeastern Brazil. Brazilian Journal of Biology 63(4):579-588.

Esteves, K. E. 1996. Feeding ecology of three Astyanax species (Characidae, Tetragonopterinae) from a floodplain lake of Mogi-Guaçú River, Paraná River Basin, Brazil. Environmental Biology of Fishes 46:83-101.

Esteves, K. E. \& Lobón-Cerviá, J. 2001. Composition and trophic structure of a fish community of a clear water Atlantic rainforest stream in southeastern Brazil. Environmental Biology of Fishes 62:429-440.

Gelwick, F. P. \& Matthews, W. J. 1996. Trophic relations of stream fishes. In: Lamberti, G. \& Hauer, R. eds. Methods in stream ecology. New York, Academic. p.475-492.

Goldstein, R. M. \& Simon, T. P. 1999. Toward a united definition of guild structure for feeding ecology of North American freshwater fishes. In: Simon, T. P. ed. Assessing the sustainability and biological integrity of water resources using fish communities. Boca Raton, CRC Press. p.123-138.

Grant, J. W. A. \& Noakes, D. L. G. 1987. A simple model of optimal territory size for drift-feeding fishes. Canadian Journal of Zoology 65:270-276.

Gregory, S. V.; Swanson, F. J.; McKee, W. A \& Cummins, K. W. 1991. An ecosystem perspective of riparian zones. BioScience 41(8):540-551.

Hölldobler, B. \& Wilson, E. O. 1990. The ants. Cambridge, Harvard University. 732p.

Hurlbert, S. H. 1978. The measurement of niche overlap and some relatives. Ecology 59(1):67-77.

Hynes, H. B. N. 1950. The food of fresh-water sticklebacks (Gasterosteus aculeatus and Pygosteus pungitius), with a review of methods used in studies of the food of fishes. Journal of Animal Ecology 19:36-58.

Keenleyside, M. H. A. 1979. Diversity and adaptation in fish behaviour. Berlin, Springer-Verlag. 208p.

Levins, R. 1968. Evolution in changing environments, some theoretical explorations. Monographs in Population Biology 2:1-120.

Lobón-Cerviá, J. \& Bennemann, S. 2000. Temporal trophic shifts and feeding diversity in two sympatric, neotropical, omnivorous fishes: Astyanax bimaculatus and Pimelodus maculatus in Rio Tibagi (Paraná, Southern Brazil). Archiv für Hydrobiologie 149(2):285-306.
Lowe-McConnell, R. H. 1987. Ecological studies in tropical fish communities. Cambridge, Cambridge University. 382p.

Mantel, N. 1967. The detection of disease clustering and generalized regression approach. Cancer Research 27:209-220.

Mazzoni, R. \& Rezende, C. F. 2003. Seasonal diet shift in a Tetragonopterinae (Osteichthyes, Characidae) from the Ubatiba river, RJ, Brazil. Brazilian Journal of Biology 63(1):69-74.

Mazzoni, R.; Fenerich-Verani, N. \& Caramaschi, E. P. 2000. Electrofishing as a sampling technique for coastal stream fish populations and communities in the southeast of Brazil. Revista Brasileira de Biologia 60(2):205-216.

NIMER, E. 1989. Climatologia do Brasil. Rio de Janeiro, IBGE. $421 \mathrm{p}$.

Ortaz, M. 2001. Diet seasonality and food overlap among fishes of the upper Orituco stream, northern Venezuela. Revista de Biología Tropical 49(1):191-197.

Prejs, A. \& Prejs, K. 1987. Feeding of tropical freshwater fishes: seasonality in resource availability and resource use. Oecologia 71:397-404.

Power, M. 1983. Grazing responses of tropical freshwater fishes to different scales of variation in their food. Environmental Biology of Fishes 9:103-115.

Reis, R. E.; Kullander, S. O. \& Ferraris Jr., C. J. 2003. Check list of the freshwater fishes of South and Central America. Porto Alegre, EDIPUCRS. 729p.

Rezende, C. F. \& Mazzoni, R. 2003. Aspectos da alimentação de Bryconamericus microcephalus (Characiformes, Tetragonopterinae) no córrego Andorinha, Ilha Grande, RJ. Biota Neotropica 3(1):1-6.

Rincón, P. A. 1999. Uso do micro-hábitat em peixes de riachos: métodos e perspectivas. In: Caramaschi, E. P.; Mazzoni, R. \& Peres-Neto, P. R. eds. Ecologia de peixes de riachos. Série Oecologia Brasiliensis, Rio de Janeiro, PPGE-UFRJ. p.23-90.

Rohlf, F. J. 2000. Software NTSYS 2.1: Numerical taxonomic and multivariate analysis system. New York, Exeter Software.

Sabino, J. 1999. Comportamento de peixes de riachos: métodos de estudo para uma abordagem naturalística. In: CARAMASCHI, E. P.; Mazzoni, R. \& Peres-Neto, P. R. eds. Ecologia de peixes de riachos. Série Oecologia Brasiliensis. Rio de Janeiro, PPGE-UFRJ. p.183-208.

Sabino, J. \& CAstro, R. M. C. 1990. Alimentação, período de atividade e distribuição espacial dos peixes de um riacho da Floresta Atlântica (Sudeste do Brasil). Revista Brasileira de Biologia 50(1):23-36.

Sabino, J. \& Zuanon, J. A. 1998. A stream fish assemblage in Central Amazonia: distribution, activity patterns and feeding behavior. Ichthyological Exploration of Freshwaters 8(3):201-210

SAzIMA, I. 1986. Similarities in feeding behaviour between some marine and freshwater fishes in two tropical communities. Journal of Fish Biology 29:53-65.

Uieda, V. S.; Buzzato, P. \& Kikuchi, R. M. 1997. Partilha de recursos alimentares em peixes de um riacho de serra no sudeste do Brasil. Anais da Academia Brasileira de Ciências 69:243-252.

Vari, R. P. \& Malabarba, L. R. 1998. Neotropical Ichthyology: an overview. In: Malabarba, L. R.; Reis, R. E.; Vari, R. P.; Lucena, Z. M. S. \& Lucena, C. A. S. eds. Phylogeny and classification of Neotropical fishes. Porto Alegre, EDIPUCRS. p.1-11.

Walker, I. 1990. Amazonian streams and small rivers. In: TuNDISI, T. M.; Tundisi, G. \& Bicudo, C. E. M. eds. Limnology in Brazil. Rio de Janeiro, ABC/SBL. p.167-193. 